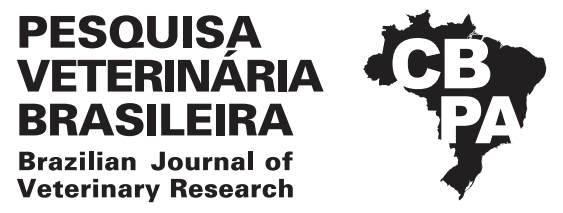

Pesq. Vet. Bras. 38(7):1250-1258, julho 2018 DOI: $10.1590 / 1678-5150-P V B-5504$

Artigo Original

Animais de Produção/Livestock Diseases

ISSN 0100-736X (Print)

ISSN 1678-5150 (Online)

\title{
Doenças podais em ovinos no estado da Bahia ${ }^{1}$
}

\author{
Vitor S. Carvalho², Marilúcia C. Santos ${ }^{3}$, José Eugênio Guimarães², \\ José Carlos Oliveira Filho ${ }^{3}$, Antônio O. Costa Neto ${ }^{4}$, Juliana T.S.A. Macêdo ${ }^{2}$ \\ e Pedro Miguel O. Pedroso ${ }^{2,5 *}$
}

\begin{abstract}
Carvalho V.S., Santos M.C., Guimarães J.E., Oliveira-Filho J.C., Costa Neto A.O., Macêdo J.T.S.A. \& Pedroso P.M.O. 2018. [Foot diseases in sheep in the state of Bahia, Brazil.] Doenças podais em ovinos no estado da Bahia. Pesquisa Veterinária Brasileira 38(7):1250-1258. Laboratório de Patologia Veterinária, Fundação Universidade de Brasília, Campus Universitário Darcy Ribeiro, Via L4 Norte s/n, Brasília, DF 70910-970, Brazil. E-mail: pedrosovet@yahoo.com.br

Foot diseases are the main causes of lameness in small ruminants, causing great impact on animal welfare and important economic losses due to depreciation and culling of affected animals. The aim of the present study was to characterize foot diseases found in eight flocks of sheep with a history of the ailment, that belonged to eight municipalities in the state of Bahia. At each visit, inspection of the flock, survey questionnaire, and characterization of diseases were performed. Altogether 600 sheep were inspected through foot examination in sick animals to characterize the injuries and record the diseases present per animal, as affected hooves and the number of affected limbs. The occurrence of foot disease was $23.3 \%(140 / 600)$ ranging from 12.8 to $55.5 \%$ per farm. Two hundred and twenty cases involving nine diseases were recorded. Foot rot was the most common disease with $51.3 \%$ of cases (113/220), 70\% corresponding to advanced and terminal stages. Interdigital dermatitis represented $27.7 \%$ of the cases, excessive hoof growth $8.6 \%$, white line disease $3.2 \%$, double soles $3.2 \%$, toe granuloma $2.3 \%$, interdigital hyperplasia $2.3 \%$, sole ulcers $0.9 \%$ and $0.4 \%$ foot abscess. The main predisposing factors involved in the genesis of diseases were the high rainfall precipitation, soil moisture conditions, pens and pastures, poor sanitary management and lack of preventive measures (footbath, quarantine, isolation and vaccination), treatment failures and low infrastructure of most properties.
\end{abstract}

INDEX TERMS: Foot diseases, lameness, sheep, foot rot.

RESUMO.- As doenças dos cascos são as principais causas de claudicação em pequenos ruminantes, causando grande impacto no bem-estar dos animais e provocando sérias perdas econômicas com depreciação e abate de animais afetados. O objetivo do presente estudo foi determinar a ocorrência e

\footnotetext{
${ }^{1}$ Recebido em 23 de junho de 2017.

Aceito para publicação em 25 de junho de 2017.

Parte da tese de doutorado do primeiro autor.

${ }^{2}$ Programa de Pós-Graduação em Ciência Animal nos Trópicos, Universidade Federal da Bahia (UFBA), Av. Adhemar de Barros 500, Ondina, Salvador, BA 40170-110, Brasil.

${ }^{3}$ Setor de Patologia Veterinária, Hospital Universitário de Medicina Veterinária, Universidade Federal do Recôncavo da Bahia (UFRB), Rua Rui Barbosa 710, Campus Universitário, Cruz das Almas, BA 44380-000, Brasil.

${ }^{4}$ Departamento de Ciências Biológicas, Universidade Estadual de Feira de Santana (UEFS), Av. Transnordestina s/n, Novo Horizonte, Feira de Santana, BA 44036-900, Brasil.

${ }^{5}$ Laboratório de Patologia Veterinária, Faculdade de Agronomia e Medicina Veterinária, Universidade de Brasília (UnB), Via L4 Norte s/n, Brasília, DF 70910-970, Brasil.*Autor para correspondência: pedrosovet@yahoo.com.br
}

caracterizar as doenças podais encontradas em oito rebanhos de ovinos com histórico de doenças podais pertencentes a oito municípios do estado da Bahia. Em cada visita, foram realizadas inspeções do rebanho, aplicação de um questionário de pesquisa e caracterização das doenças. No total, 600 ovinos foram inspecionados, realizando exame do casco em animais doentes para caracterizar as lesões, registrar a quantidade de doenças presentes por animal, o casco afetado e a quantidade de membros afetados. A ocorrência de doença de casco foi de $23,3 \%$ (140/600) variando de 12,8 a $55,5 \%$ por fazenda. Duzentos e vinte casos envolvendo nove doenças foram registrados. A pododermatite infecciosa foi a doença mais comum com $51,3 \%$ dos casos (113/220), 70\% correspondente aos estágios avançados e terminais. A dermatite interdigital representou $27,7 \%$ dos casos; crescimento excessivo dos cascos $8,6 \%$; doença da linha branca $3,2 \%$; sola dupla $3,2 \%$; granuloma do dígito 2,3\%; hiperplasia interdigital 2,3\%; úlcera de sola $0,9 \%$ e $0,4 \%$ de abcesso de pé. Os principais fatores predisponentes envolvidos na ocorrência das doenças foram 
a alta precipitação pluviométrica, condições de umidade do solo, currais e pastagens, manejo sanitário deficiente e falta de medidas preventivas (pedilúvio, quarentena, isolamento e vacinação), falhas de tratamento e baixa infra-estrutura da maioria das propriedades.

TERMOS DE INDEXAÇÃO: Doenças podais, claudicação, ovinos, pododermatite infecciosa.

\section{INTRODUÇÃO}

Claudicação resulta de uma condição dolorosa e estressante, com um grande impacto no bem-estar, saúde e produção animal. Em pequenos ruminantes, lesões no casco ou na pele circundante são as principais causas de claudicação e a dor pode facilmente perturbar o frágil equilíbrio da saúde dos animais e levar a uma queda imediata no desempenho (Maes \& Opsomer 2013). Dermatite interdigital, pododermatite infecciosa, abscesso do pé, úlceras de sola, doença da linha branca, traumas ou ferimentos por corpos estranhos, lesões granulomatosas e crescimento excessivo dos cascos são exemplos de doenças que frequentemente levam a dores e lesões nos dígitos de caprinos e ovinos. Dentre essas enfermidades, a pododermatite infecciosa ocupa lugar de destaque pela frequência e impactos nos rebanhos (Aguiar et al. 2011).

As enfermidades podais são comumente relatadas por produtores, sendo menos frequentes, apenas, que as endoparasitoses e distúrbios reprodutivos (Pinheiro et al. 2000). Embora frequentes, existem muitas falhas no diagnóstico que provocam uma conduta errônea diante de surtos, dificultando o sucesso dos tratamentos implementados. No caso de claudicação no rebanho, é essencial examinar um número suficiente de animais para se ter uma imagem clara do tipo de doença envolvida. Muitas vezes, mais de um tipo de alteração está presente e casos iniciais ou crônicos podem confundir o diagnóstico (Winter 2008). No Brasil, ainda são escassas as pesquisas sobre a ocorrência e diagnóstico dessas doenças em ovinos. $\mathrm{O}$ objetivo deste trabalho foi determinar a ocorrência e caracterizar as doenças podais encontradas em criações de ovinos de diferentes municípios do estado da Bahia.

\section{MATERIAL E MÉTODOS}

O estudo foi conduzido de 2013 a 2015 em oito fazendas de criação semi-intensiva ou extensiva de ovinos, de oito municípios do estado da Bahia (São Gonçalo dos Campos, Cachoeira, Entre Rios, Cruz das almas, Muritiba, Candeias, Mata de São João e São Felipe), pertencentes a quatro regiões do estado (Recôncavo, Litoral Norte/Agreste Baiano, Portal do Sertão e Região Metropolitana de Salvador). Os ovinos possuíam diferentes faixas etárias e com exceção dos reprodutores e matrizes eram mestiços da raça Santa Inês com Dorper. Todas as fazendas possuíam histórico de doenças podais e foram escolhidas por indicações da Associação de Criadores de Caprinos e Ovinos do Estado da Bahia (ACCOBA), médicos veterinários de campo e de lojas agropecuárias.

No início de cada visita clínica, foi aplicado um questionário para investigar fatores predisponentes e caracterizar aspectos relacionados ao sistema de criação, infraestrutura, manejo sanitário, surgimento (hipótese), ocorrência, controle e tratamento de doenças de casco. 0 índice pluviométrico das regiões visitadas foi obtido através de consulta ao banco de dados do setor de monitoramento do Instituto de Meio Ambiente e Recursos Hídricos (INEMA). Após o questionário, foi realizada inspeção no rebanho de cada propriedade para identificação de animais com claudicação ou outros sinais de enfermidades podais. Ao todo, 600 ovinos foram observados. A ocorrência de animais doentes foi registrada por fazenda e pelo rebanho total.

Os animais foram contidos para exame podal, sendo cada casco lavado e inspecionado, catalogando-se as enfermidades em uma ficha própria para doenças digitais, com a identificação do casco acometido e caracterização das lesões. Em cada animal foi registrada a quantidade de doenças presentes, o membro afetado e a quantidade de membros acometidos. Os casos de pododermatite infecciosa foram classificados de acordo com a gravidade da doença em estágio inicial (dermatite interdigital severa e necrose na região axial da sola e talão), avançado (necrose no talão e em toda extensão da sola atingindo a margem abaxial) ou terminal (extensão da necrose para a muralha com descolamento de todo o casco). Nos animais que exibiam apenas inflamação leve a moderada da pele interdigital sem sinais de descolamento ou necrose deste ou de tecidos vizinhos, a lesão foi caracterizada como dermatite interdigital. A análise estatística foi realizada no programa Statística v.7, utilizando o Teste Z para detectar diferenças entre duas proporções, com 95\% de confiança $(p<0,05)$.

\section{RESULTADOS}

De acordo com o questionário, os principais fatores predisponentes observados foram: alto índice pluviométrico (143-258mm) no período de maior ocorrência das doenças na região; ausência de limpeza e baixa higiene nos apriscos e no veículo de transporte (quando presente); alta umidade nos currais e pastos; ausência de medidas profiláticas (pedilúvio, quarentena, isolamento e vacinação); ausência de conhecimento da doença ou experiência com ovinos; manutenção de portadores crônicos e falhas no tratamento. Metade das propriedades realizava casqueamento, porém sem uma frequência pré-estabelecida. Em quatro propriedades de criação mista houve queixa de extensão das doenças podais para os caprinos, com relato de casos graves de "podridão dos cascos". Em todas as propriedades foram relatados prejuízos econômicos relacionados à perda de peso, queda dos índices reprodutivos, descarte de animais e altos custos anuais com mão de obra e tratamento.

Quanto ao surgimento da pododermatite nas propriedades, a principal hipótese escolhida pelos criadores foi que a doença começou após a introdução ou aquisição de um ovino doente no rebanho. Em todas as propriedades, exceto do município de Cruz das Almas, cujo início da criação de ovinos era recente (6 meses), houve relato de ocorrência anterior de pododermatite em outros anos ou em fazendas vizinhas e de presença de casos durante todo o ano, sendo os surtos na época de chuvas. Um dos produtores informou que há 20 anos convive com a doença.

O histórico de índice pluviométrico das regiões analisadas é alto, variando entre 800 a $2.000 \mathrm{~mm}$ anuais. No período do estudo (2013-2015), a precipitação mostrou-se concentrada entre os meses de abril a agosto, sendo os meses de maio, junho e julho os que registraram a maior pluviosidade mensal do ano, variando de 143mm (julho/2013) até 258mm (maio/2014). 
Dos 600 ovinos observados, 140 apresentaram doenças podais, obtendo-se a ocorrência de $23,3 \%$, variando de $12,8 \%$ até $55,5 \%$ entre as propriedades (Quadro 1). Em cada fazenda foi possível observar ao menos três tipos de enfermidades podais. Ao todo, foram registrados 220 casos envolvendo nove doenças (Quadro 2). A maioria dos animais acometidos apresentavam-se com postura ajoelhada (apoio sobre os carpos) devido à dor provocada por lesões podais nos membros torácicos (Fig.1). A doença mais diagnosticada foi a pododermatite infecciosa com 51,3\% (113/220) dos casos, seguida da dermatite interdigital com 27,7\% (61/220) e crescimento excessivo do casco com 8,6\% (19/220).

Dentre os 113 registros de pododermatite infecciosa, 15,4\% correspondiam ao estágio inicial (Fig.2A), 27,7\% ao estágio avançado (Fig.2B) e 8,1\% ao estágio terminal da doença (Fig.2C). Na dermatite interdigital, essa foi caracterizada por lesão úmida de coloração esbranquiçada, com sensibilidade, inflamação, erosão e alopecia local (Fig.3). Crescimento excessivo do casco, deformidade caracterizada por crescimento exacerbado e irregular das pinças com unhas laterais encurvando-se dorsomedialmente (Fig.4). Observou-se também crescimento excessivo e irregular das pinças encurvando-se dorsalmente ou plantarmente e crescimento da face abaxial da muralha plantarmente, atingindo a face axial. Doença da linha branca $(3,1 \%)$, caso severo com descolamento da muralha e exposição das lâminas (Fig.5). A doença da linha branca variou de pequenas lesões discretas isoladas na zona abaxial ou axial, até uma grande perda da parede do casco com exposição das lâminas; sola dupla $(3,1 \%)$, caracterizado por espaço com formação de uma segunda sola em ambas as unhas (Fig.6). Granuloma do dígito $(2,2 \%)$, caracterizado pela presença de tecido de granulação e fácil sangramento localizado na região da pinça da unha

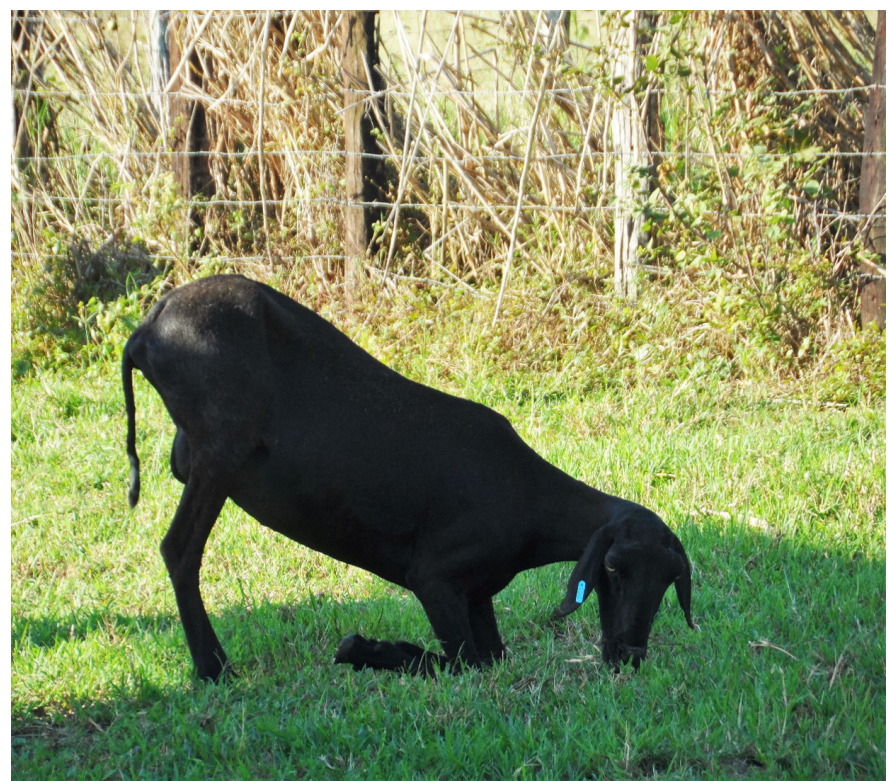

Fig.1. Animal exibindo postura ajoelhada (apoio sobre os carpos) devido à dor provocada por lesões podais nos membros torácicos.

Quadro 1. Ocorrência de ovinos com doenças podais, nos anos de 2013 a 2015, em rebanhos provenientes de oito municípios baianos

\begin{tabular}{lccc}
\hline \multicolumn{1}{c}{ Propriedades } & № de animais avaliados & № de animais doentes & Ocorrência (\%) \\
\hline Entre Rios & 230 & 44 & 19,13 \\
Cachoeira & 70 & 17 & 24,29 \\
São Gonçalo dos Campos & 80 & 15 & 18,75 \\
Cruz das Almas & 28 & 14 & 50,00 \\
Muritiba & 60 & 14 & 23,33 \\
Candeias & 18 & 10 & 55,56 \\
Mata de São João & 70 & 9 & 12,86 \\
São Felipe & 44 & 17 & 38,64 \\
Total & 600 & 140 & 23,33
\end{tabular}

Quadro 2. Classificação das doenças podais observadas, de 2013 a 2015, em ovinos de rebanhos provenientes de oito municípios baianos

\begin{tabular}{|c|c|}
\hline Doença podal & Número de casos (\%) \\
\hline Pododermatite infecciosa & $113(51,36 \%)$ \\
\hline Estágio inicial & $34(15,45 \%)$ \\
\hline Estágio avançado & $61(27,73 \%)$ \\
\hline Estágio terminal & $18(8,18 \%)$ \\
\hline Dermatite interdigital & $61(27,73 \%)$ \\
\hline Crescimento excessivo & $19(8,64 \%)$ \\
\hline Doença da linha branca & $7(3,18 \%)$ \\
\hline Sola dupla & $7(3,18 \%)$ \\
\hline Granuloma de dígito & $5(2,27 \%)$ \\
\hline Hiperplasia interdigital & $5(2,27 \%)$ \\
\hline Úlcera de sola & $2(0,91 \%)$ \\
\hline Abscesso de casco & $1(0,45 \%)$ \\
\hline TOTAL & $220(100 \%)$ \\
\hline
\end{tabular}




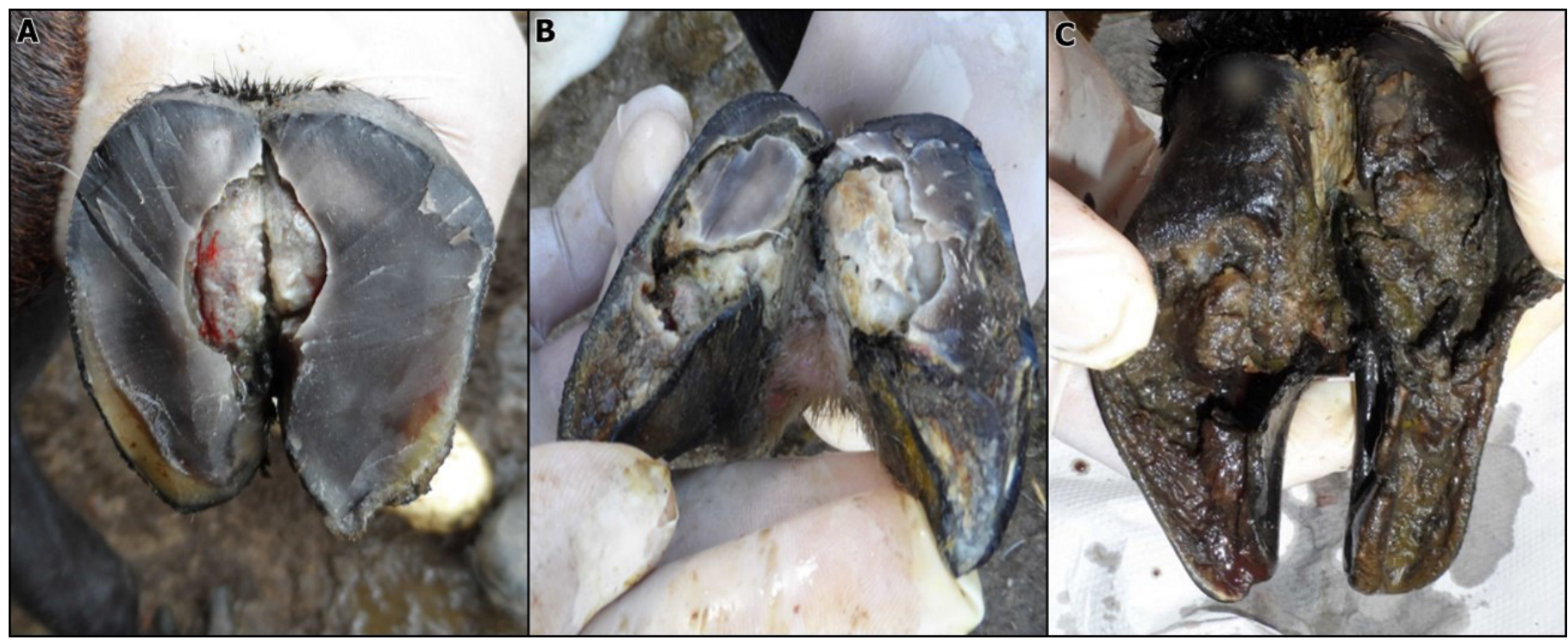

Fig.2. Diferentes estágios observados nos casos de pododermatite infecciosa em ovinos no estado da Bahia. (A) Estágio inicial: dermatite interdigital severa associada a necrose na região axial da sola e talão. (B) Estágio avançado: necrose no talão e em toda extensão da sola atingindo a margem abaxial. (C) Estágio terminal: extensão da necrose para a muralha e com descolamento de todo o casco.

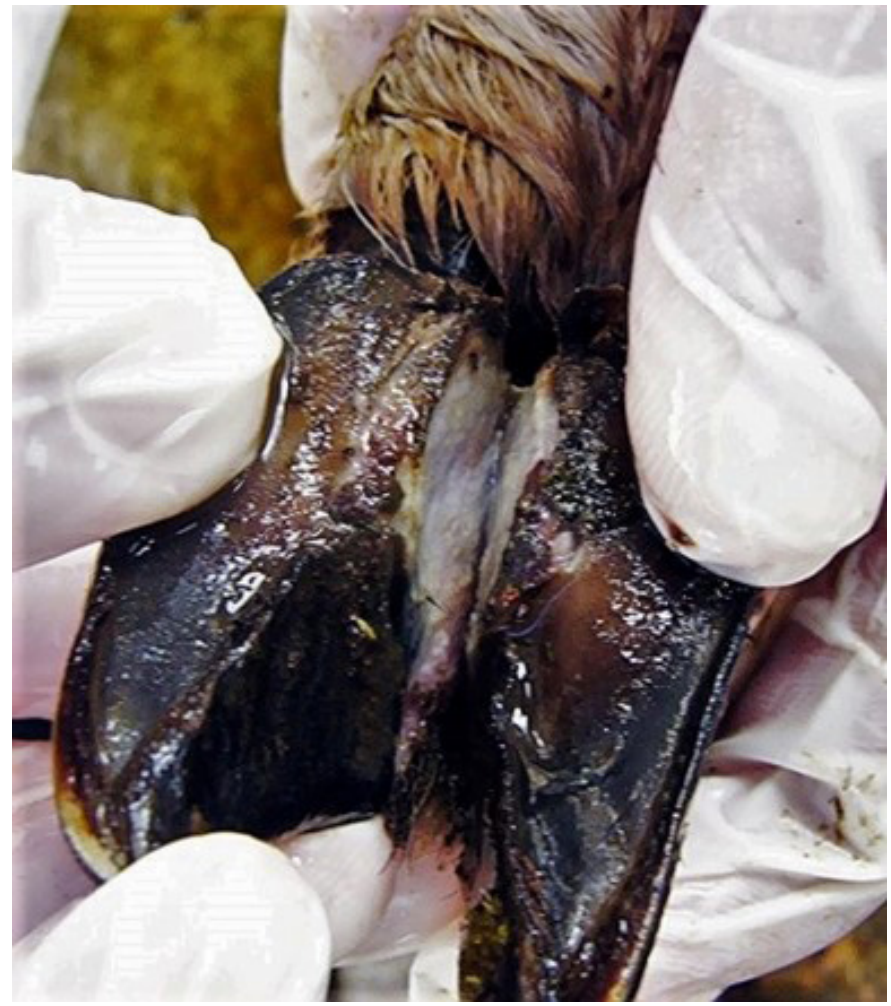

Fig.3. Dermatite interdigital caracterizada por lesão úmida de coloração esbranquiçada, com sensibilidade, inflamação, erosão e alopecia local.

medial (Fig.7). As lesões granulomatosas exibiam protrusão do tecido na região da pinça, sola ou interdigital, com muito sangramento e provocando claudicação severa. Hiperplasia interdigital (2,2\%), apresentando crescimento de tecido fibroso, de baixa vascularização no espaço interdigital, com erosão na face plantar (Fig.8). Úlcera de sola (0,9\%), lesão de formato circular em cicatrização entre a sola e o talão da unha lateral e abscesso de casco, caracterizado por aumento de volume da

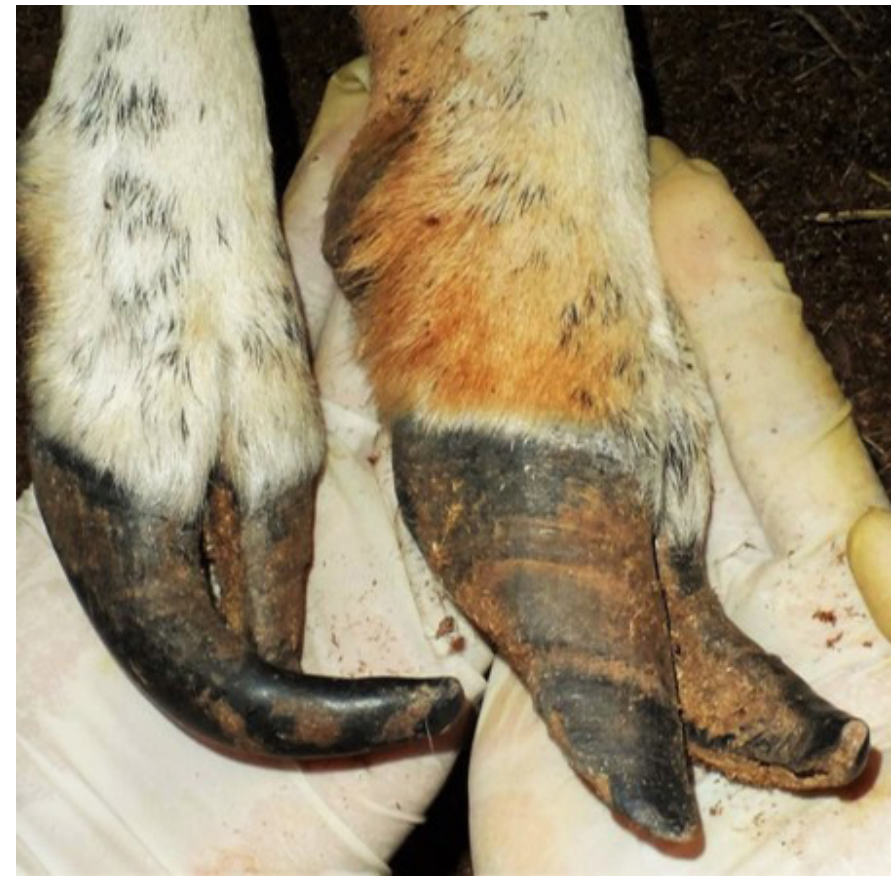

Fig.4. Crescimento excessivo de casco. Deformidade caracterizada por crescimento exacerbado e irregular das pinças com unhas laterais encurvando-se dorsomedialmente.

unha medial associado à lesão supurativa e necrótica drenando secreção purulenta na região da sola e pinça (Fig.9). 0 único caso de abscesso de casco (0,4\%) (Fig.10) foi observado em uma ovelha, no membro pélvico esquerdo, associado à presença concomitante de pododermatite. Os ovinos doentes apresentavam baixo escore corporal, apatia e sinais de dor que variava de branda a severa e claudicação que variava de discreta a acentuada.

Quanto à distribuição das doenças podais nos animais, a maior ocorrência observada nos cascos dos membros torácicos $(53,5 \%)$ não diferiu significativamente dos pélvicos $(46,5 \%)$. 


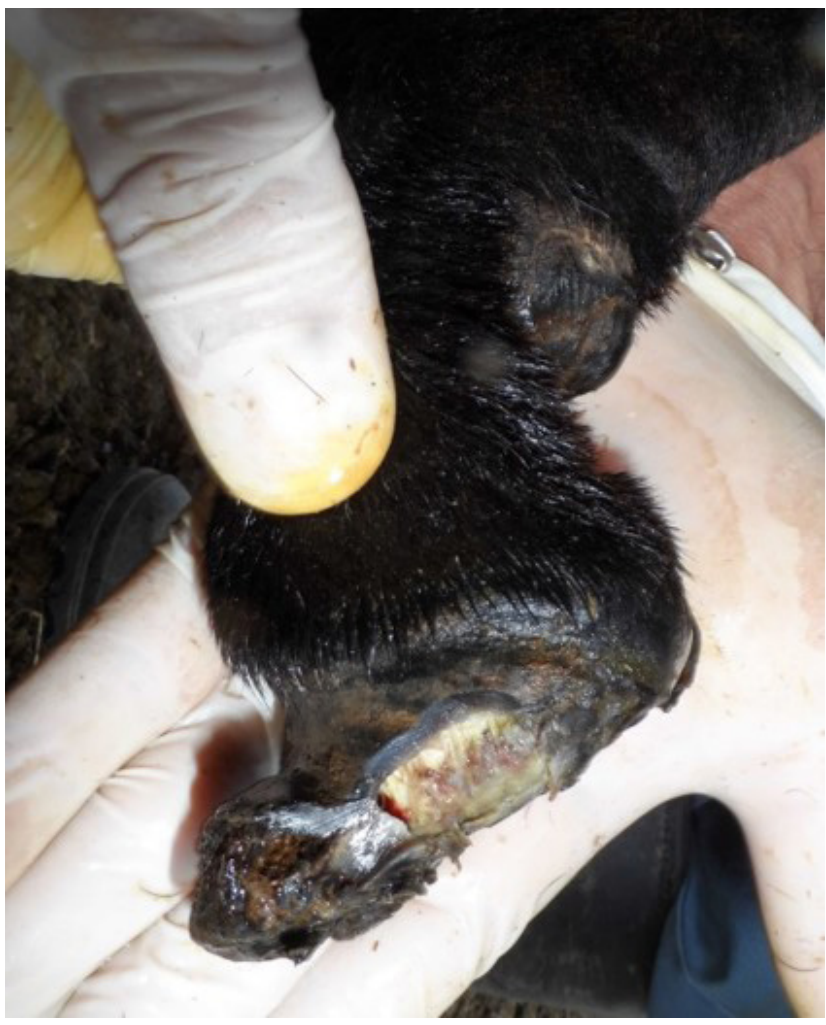

Fig.5. Doença da linha branca. Caso severo com descolamento da muralha e exposição das lâminas.

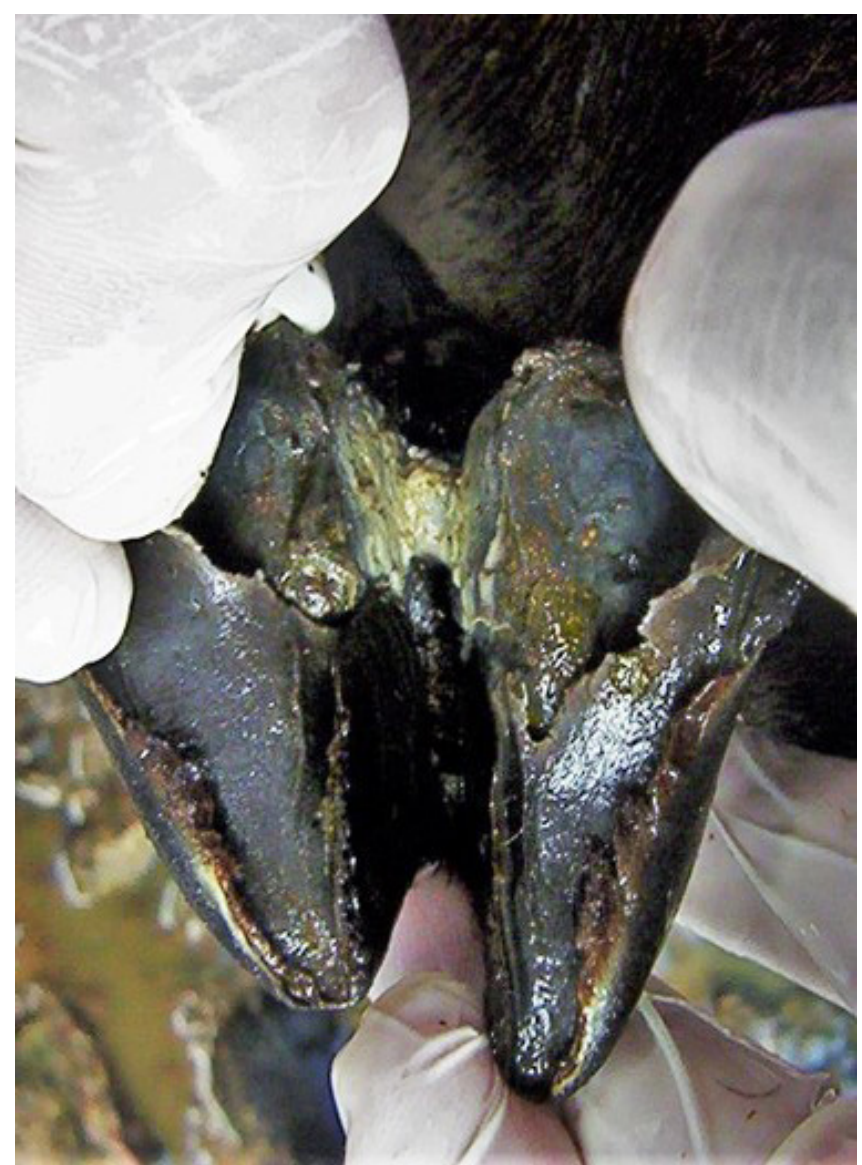

Fig.6. Sola dupla. Espaço com formação de uma segunda sola em ambas as unhas.

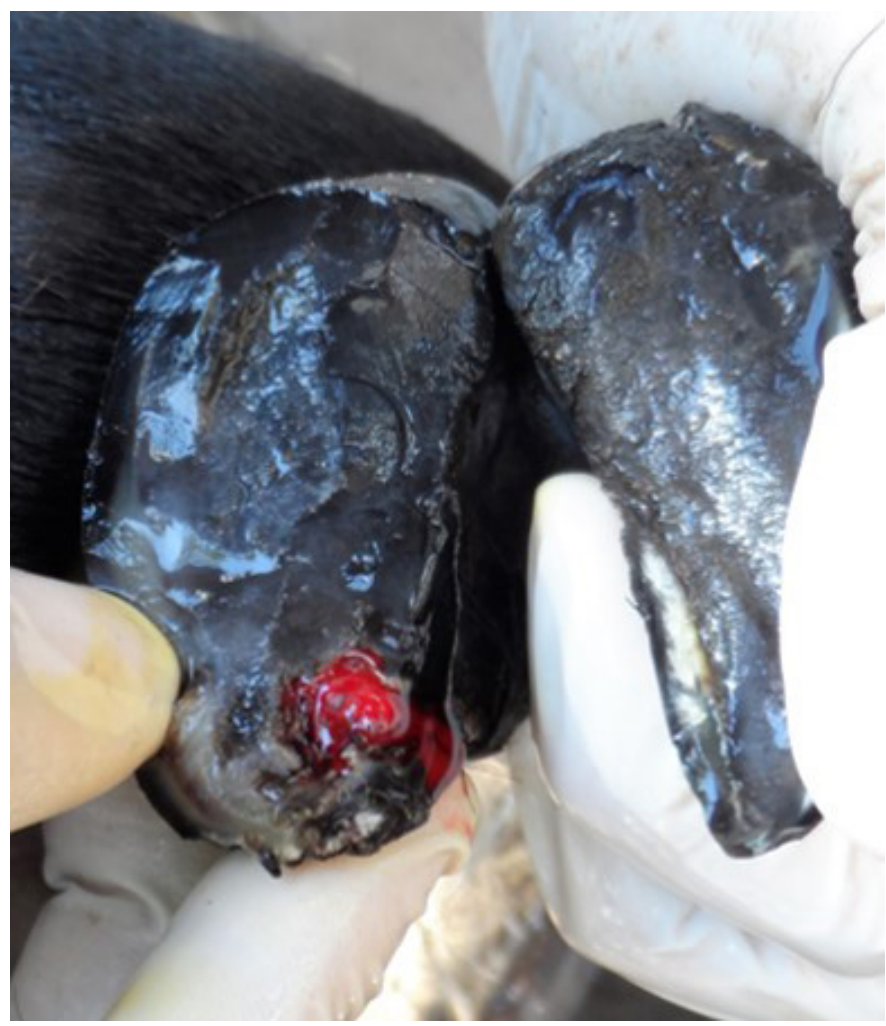

Fig.7. Granuloma de dígito. Presença de tecido de granulação e fácil sangramento localizado na região da pinça da unha medial.

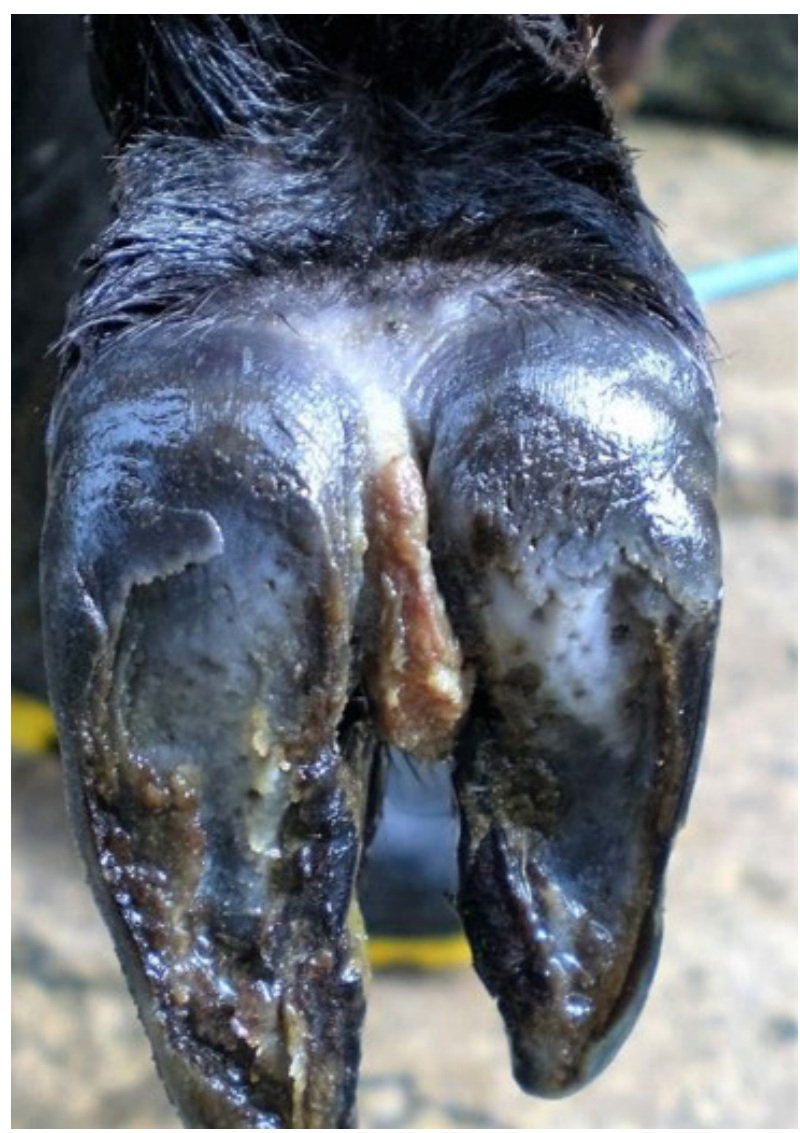

Fig.8. Hiperplasia interdigital. Crescimento de tecido fibroso, de baixa vascularização no espaço interdigital, com erosão na face plantar. 
Quanto à quantidade de membros acometidos (Quadro 3) e quantidade de doenças por animal (Quadro 4), 80\% dos animais exibiram lesões em mais de um membro e $46,4 \%$ foram acometidos por mais de um tipo de doença. Na maioria destes casos a pododermatite ou a dermatite interdigital era a doença primária ocorrendo em associação com as outras de menor frequência.

\section{DISCUSSÃO}

O presente trabalho registrou uma alta ocorrência $(23,3 \%)$ envolvendo múltiplas doenças no mesmo rebanho ou animal. Segundo a literatura, um índice de claudicação por doenças

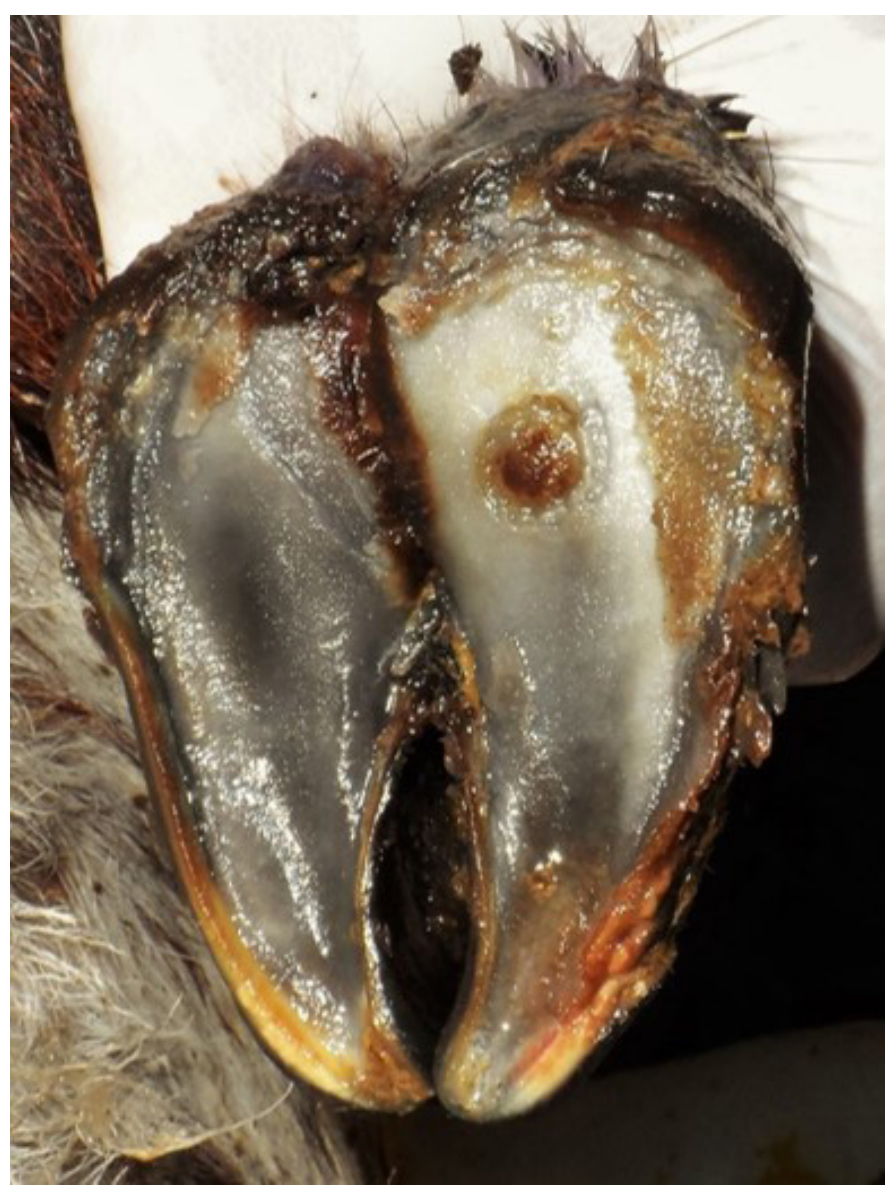

Fig. 9. Úlcera de sola. Lesão de formato circular em cicatrização entre a sola e o talão da unha lateral.

Quadro 3. Número de membros acometidos por doenças podais em ovinos provenientes de oito municípios baianos, no período de 2013 a 2015

\begin{tabular}{cl}
\hline $\begin{array}{c}\text { Quantidade de membros } \\
\text { acometidos }\end{array}$ & $\begin{array}{c}\text { Número de ovinos } \% \\
\text { (n⿳o ovinos/total) }\end{array}$ \\
\hline 1 & $20,0 \%(28 / 140) \mathrm{a}$ \\
2 & $32,1 \%(45 / 140) \mathrm{b}$ \\
3 & $20,7 \%(29 / 140) \mathrm{a}$ \\
4 & $27,2 \%(38 / 140) \mathrm{a}, \mathrm{b}$
\end{tabular}

*Letrasiguais na mesma coluna os percentuais não diferem significativamente pelo teste Z para diferença entre proporções ( $p>0,05)$. podais superiores a 5\% em um rebanho ovino já é preocupante, devendo-se estabelecer as causas e tomar providencias para o tratamento (O’Leary 2014). Dados próximos ao presente estudo foram encontrados em surtos de doenças podais no estado da Paraíba, onde a ocorrência em ovinos foi de 19,4\% (170/876), encontrando-se nessa espécie as mesmas doenças aqui relatadas com exceção de granuloma do dígito, hiperplasia interdigital e sola dupla (Aguiar et al. 2011). Valores inferiores foram relatados em Minas Gerais, com 11\% (Morão et al. 2015), em rebanhos no Rio Grande do Sul, com 14\% (Silveira et al. 2016) e em pesquisas em outros países como $6,6 \%$ no Uruguai, de 8 a $10 \%$ nos países do Reino Unido e de $12 \%$ na Índia (Bonino et al. 2000, Olechnowicz \& Jáskowski 2011, Rather et al. 2011). Pesquisas recentes relataram uma diminuição da prevalência de doenças podais na Inglaterra entre 2004 e 2013 , de $10,6 \%$ para $4,9 \%$, devido à implantação e execução de medidas de controle durante o período (Winter et al. 2015).

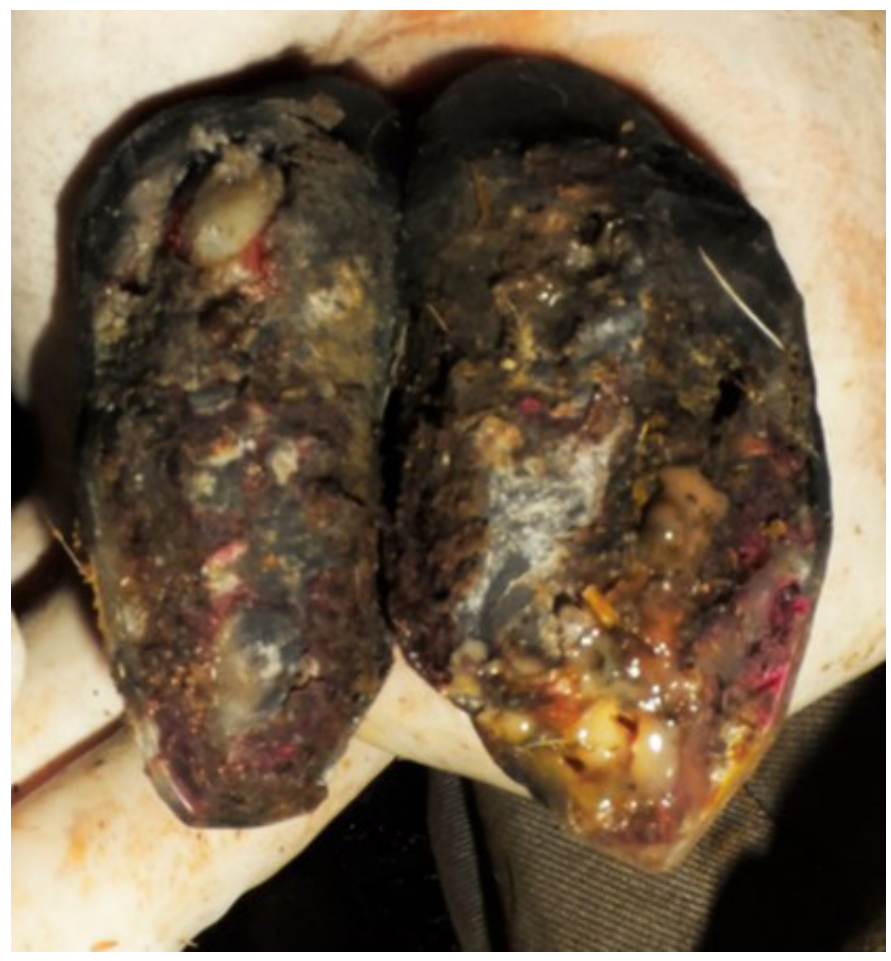

Fig.10. Abscesso de casco. Aumento de volume da unha medial associado à lesão supurativa e necrótica drenando secreção purulenta na região da sola e pinça.

Quadro 4. Quantitativo de doenças podais por animal, em ovinos provenientes de oito municípios baianos, no período de 2013 a 2015

\begin{tabular}{cc}
\hline $\begin{array}{c}\text { Quantidade de doenças } \\
\text { por animal }\end{array}$ & $\begin{array}{c}\text { Número de ovinos } \% \\
\text { (no ovinos/total) }\end{array}$ \\
\hline 1 tipo & $53,6 \%(75 / 140) \mathrm{a}^{*}$ \\
2 tipos & $35,7 \%(50 / 140) \mathrm{b}$ \\
3 tipos & $9,3 \%(13 / 140) \mathrm{c}$ \\
4 tipos & $1,4 \%(2 / 140) \mathrm{d}$
\end{tabular}

*Letrasiguais namesma coluna os percentuais não diferem significativamente pelo teste $\mathrm{Z}$ para diferença entre proporções $(\mathrm{p}>0,05)$. 
De acordo com os resultados apresentados, a pododermatite infecciosa e a dermatite interdigital ocuparam posição de destaque dentre as doenças podais identificadas, representando $79 \%$ dos casos. Estes dados concordam com as observações de outros autores que afirmam que a pododermatite e a dermatite interdigital são as duas maiores causas de claudicação em ovinos, representando de 80 a $90 \%$ dos casos de doenças podais (Olechnowicz \& Jáskowski 2011, Winter et al. 2015). Na Paraíba, foi relatado que associações de dermatite interdigital e pododermatite infecciosa foram responsáveis por $71 \%$ das lesões diagnosticadas em ovinos (Aguiar et al. 2011). Em estudo de um surto de claudicação em um rebanho no estado da Bahia, foi observado um índice de 38,95\% de ovinos com pododermatite (Cavalcanti et al. 2004). Já no estado de São Paulo, foi descrito um surto de pododermatite que acometeu 100\% de um rebanho com 400 animais (Rodrigues et al. 2001). A pododermatite também foi descrita como a afecção podal de maior ocorrência no serviço de clínica de pequenos ruminantes da Universidade de São Paulo, com 60\% dos casos podais atendidos (Gargano et al. 2013).

A claudicação foi observada em todos os animais doentes, porém seu grau variava conforme o tipo, gravidade da doença e quantidade de cascos afetados. Dentre os casos de pododermatite infecciosa, 70\% corresponderam a estágios avançados e terminais da doença indicando que a forma virulenta era a prevalente nos rebanhos consultados. A classificação das lesões de pododermatite também foi realizada em um surto no interior de São Paulo, observando a forma severa em 85\% dos ovinos do rebanho (Rodrigues et al. 2001). A presença da forma virulenta nas propriedades do presente estudo também pode ser sugerida pelo relato, durante questionário, de acometimento de vários caprinos nas quatro propriedades que possuíam criação consorciada com os ovinos. É descrito que os caprinos são mais resistentes que ovinos, pois o extrato córneo da pele interdigital é mais espesso, o que dificulta a invasão bacteriana nos animais sujeitos à infecção e à manifestação de lesões mais graves (Ghimire et al. 1999).

0 crescimento excessivo do casco representou a terceira doença mais diagnosticada, porém com somente 8,6\% dos casos. Este valor pode estar ligado ao fato que metade das fazendas consultadas realizava o casqueamento, mesmo que esporádico. Valores extremos de ocorrência podem ser encontrados na literatura variando desde 0,5\% (Aguiar et al. 2011) a 90\% (Tadich \& Hernandez 2000). 0 crescimento excessivo dos cascos pode levar a sérios problemas devido a: acúmulo de solo e matéria orgânica úmida, predispondo à pododermatite, ou matéria inorgânica ressecada (como pedras) gerando lesões traumáticas; deformação da unha com angulação incorreta ou torção das estruturas internas impedindo o apoio correto ao solo e possibilidade de fissura ou ruptura da muralha com exposição das lâminas sensitivas (Smith et al. 2014).

Doença da linha branca, sola dupla, granuloma do dígito, hiperplasia interdigital, úlcera de sola e abscesso de casco foram as enfermidades de menor ocorrência. A maioria destes casos estavam ligados à presença simultânea de pododermatite em diferentes estágios, concordando com dados da literatura (Riet-Correa 2007, Hodgkinson 2010, Aguiar et al. 2011). 0 defeito da linha branca variou desde discretas lesões, suspeitas de serem consequência de traumas ou casqueamento agressivo, até gravíssimas, nos casos provocados por pododermatite avançada ou terminal. Levantamentos realizados em São Paulo (Gargano et al. 2013) e Paraíba (Aguiar et al. 2011) com pequenos ruminantes apontaram que essa doença também foi pouco frequente, representando $2 \%$ e 3,95\% dos animais consultados. Já estudos em ovinos nos países do Reino Unido apontam que lesões da linha branca são extremamente comuns (Winter 2008).

Os casos de sola dupla e a hiperplasia interdigital encontrados neste estudo estavam associados a casos de pododermatite $\mathrm{e}$ dermatite interdigital, respectivamente. Essas doenças também foram encontradas com baixa ocorrência em rebanhos ovinos chilenos (Tadich \& Hernandez 2000), com prevalência de 1,7\% para sola dupla e $0,4 \%$ para hiperplasia interdigital. A úlcera de sola é rara em pequenos ruminantes e já foi descrita no Brasil, podendo ocorrer consequente a progressões de lesões da pododermatite ou ser provocada por traumas na sola (Aguiar et al. 2011, Olechnowicz \& Jáskowski 2011). Um dos casos registrados neste estudo ocorreu isolado entre a sola e o talão de um casco sem pododermatite e dessa forma suspeitou-se de traumatismo.

Para lesões granulomatosas, são descritas as mesmas causas da úlcera de sola, com muitos casos ocorrendo devido à casqueamento agressivo ou posteriormente à formação dessas úlceras. Granulomas representam riscos secundários já que a lesão pode servir como reservatório para persistência do Dichelobacter nodosus no rebanho (Hodgkinson 2010). Os casos de granuloma do dígito do estudo foram observados associados ou não a pododermatite e ocorreram nas quatro propriedades que realizavam casqueamento preventivo.

0 único registro de abscesso de casco do estudo concorda com observações da literatura que afirmam que essa infecção é uma complicação da dermatite interdigital, acometendo principalmente ovinos adultos, com baixa morbidade e manifestando-se na maioria das vezes em um só membro, envolvendo especialmente os pélvicos (Riet-Correa 2007).

De acordo com a distribuição observada nos animais do estudo, apesar da maior frequência de acometimento dos cascos dos membros torácicos não houve diferença significativa com os pélvicos. Uma maior ocorrência nos membros torácicos foi descrita em pesquisas que justificam que os cascos anteriores suportam cerca de 60-65\% do peso corporal e auxiliam os pélvicos na propulsão do corpo, estando mais propensos a lesões por trauma e abalos que os pélvicos (Bokko et al. 2003, Carvalho et al. 2012, Gargano et al. 2013, Morão et al. 2015). A presença de lesões nos membros torácicos faz com que os animais andem de joelhos, sendo uma alteração frequentemente observada durante as visitas clínicas deste estudo.

Quanto à quantidade de membros acometidos, um número expressivo de animais (27\%) chamou atenção por exibir lesões nos quatro membros. Em estudo anterior na Bahia, também foi observado uma grande quantidade de animais com lesões nos quatro membros (20\%) (Carvalho et al. 2012). Valores extremos já foram relatados no estado de São Paulo, onde em $100 \%$ dos animais de um rebanho ovino, mais de um membro foi acometido e em $87 \%$ os quatro membros foram atingidos (Rodrigues et al. 2001).

Avaliando-se a quantidade de doenças por animal, observou-se que uma grande parcela de animais $(46,4 \%)$ foi acometido por mais de um tipo de doença, chegando a encontrar até quatro tipos de doenças podais no mesmo animal. Em levantamento de lesões podais em ovinos no Chile, 
foi relatado que $69 \%$ dos ovinos estudados apresentavam múltiplas lesões e que, essas associações potencializavam a gravidade dos casos, gerando dificuldade na marcha e na alimentação do animal (Tadich \& Hernandez 2000). Muitos animais do presente estudo exibiam lesões irreversíveis ou de difícil tratamento e já estavam selecionados para descarte. Percebeu-se, em todas as propriedades, que a maioria dos prejuízos econômicos citados pelos produtores, incluindo essas por descarte involuntário e tratamentos, poderiam ter sido evitados caso existisse o reconhecimento ou diagnóstico precoce das doenças.

O questionário aplicado em cada visita buscou identificar fatores predisponentes que contribuíram para o aparecimento das doenças ou maior prevalência de alguma delas. A falta de conhecimento mais profundo da doença pelo produtor ou a inexperiência com ovinos por tratadores impediu um reconhecimento precoce das doenças, gerando diversas falhas no tratamento e permitindo a entrada de animais doentes e a manutenção de portadores crônicos no rebanho. Todos estes fatos são contrários aos preceitos citados para evitar e controlar as doenças podais em um rebanho (O'Leary 2014). As perdas econômicas relatadas nas propriedades visitadas condizem com as observações de outros trabalhos, sobre custos e impactos econômicos relativos à presença de afecções podais em ovinos (Bonino et al. 2000, Hodgkinson 2010). Segundo um estudo na Irlanda cada ovelha doente gera um custo de cerca de 18 dólares para o produtor (Lovatt 2015).

A associação do índice pluviométrico de cada região com os fatores relacionados às condições de umidade do solo, currais e pastos, manejo sanitário deficiente, ausência de medidas profiláticas (pedilúvio, quarentena, isolamento e vacinação) e a infraestrutura de cada propriedade contribuíram para o número de ovinos enfermos no estudo. Observações semelhantes já foram relatadas em trabalhos realizados na Austrália (Abbott \& Egerton 2003), quando avaliaram rebanhos mantidos em criatórios de características climáticas diversas, e em diferentes estados brasileiros (Rodrigues et al. 2001, Ribeiro 2010, Aguiar et al. 2011, Carvalho et al. 2012). Essas pesquisas também concluíram que a exposição dos cascos por longos períodos em ambientes e pastagens úmidas e em condições adversas predispõe a infecção e a transmissão das doenças entre os animais.

Acredita-se que as condições ambientais observadas durante o período do presente estudo favoreceram a difusão das enfermidades gerando a alta ocorrência encontrada. Na Austrália, a transmissão da pododermatite está associada com chuvas e pastagens luxuriantes. Nesse país, as áreas endêmicas demonstram uma precipitação anual de 500-600 mm e o início de surtos necessita de precipitações continuadas de $50 \mathrm{~mm}$, por dois a três meses (Ribeiro 2010). Os valores anuais e mensais das regiões visitadas no presente estudo são bem superiores aos encontrados nas áreas endêmicas da Austrália. 0 índice pluviométrico no período analisado mostrou-se mais elevado entre os meses de abril a agosto (INEMA 2016) que coincide com o chamado "período de chuvas" descrito por alta ocorrência ou surtos de claudicação dos ovinos nas propriedades consultadas.

A hipótese escolhida pelos proprietários para explicar o surgimento da pododermatite nas propriedades visitadas (aquisição de um animal portador) é embasada por diversos trabalhos que demonstram que a bactéria responsável pelos surtos (Dichelobacter nodosus) sobrevive poucos dias fora do casco, necessitando de animais portadores (ovinos ou caprinos) para manter ou iniciar a transmissão em um rebanho (Ghimire et al. 1999, Ribeiro 2010, Raadsma \& Egerton 2013). A ausência de quarentena nas propriedades visitadas também reforça essa hipótese.

\section{CONCLUSÕES}

A elevada ocorrência de lesões digitais em ovinos encontrada no estudo revela que as doenças podais representam um grave problema nas regiões visitadas e que as características ambientais e de manejo podem ser consideradas como fator determinante no estabelecimento e manutenção dessas enfermidades.

Os resultados demonstram que diversas afecções podais de ovinos podem estar presentes no mesmo rebanho ou animal e que a pododermatite infecciosa é a doença podal mais frequente, causando graves lesões nos animais.

A falta de conhecimento dos produtores a respeito das doenças podais contribui ativamente para a alta ocorrência e manutenção de ovinos enfermos nos rebanhos.

\section{REFERÊNCIAS}

Abbott K.A. \& Egerton J.R. 2003. Effect of climatic region on the clinical expression of footrot of lesser clinical severity (intermediate footrot) in sheep. Aust. Vet. J. 81(12):756-762. <http://dx.doi.org/10.1111/j.1751-0813.2003. tb14609.x><PMid:15080488>

Aguiar G.M.N., Simões S.V.D., Silva T.R., Assis A.C.O., Medeiros J.M.A., Garino Júnior F. \& Riet-Correa F. 2011. Footrot and other foot diseases of goat and sheep in the semiarid region of northeastern Brazil. Pesq. Vet. Bras. 31(10):879-884. <http://dx.doi.org/10.1590/S0100-736X2011001000008>

Bokko B.P., Adamu S.S. \& Mohammed A. 2003. Limb conditions that predispose sheep to lameness in the arid zone of Nigeria. Small Rumin. Res. 47(2):165169. <http://dx.doi.org/10.1016/S0921-4488(02)00254-7>

Bonino J., Casaretto A., Mederos A., Ferreira G. \& Gil A. 2000. Relevamiento epidemiológico de la prevalencia de footrot en ovinos del Uruguay. Prod. Ovina (13):9-25.

Carvalho V.S., Araújo B.R., Vasconcelos T.C., Chiminazzo C., Costa Neto A.O., Ayres M.C.C., Guimarães J.E. \& Costa J.N. 2012. Evolução clínica e avaliação de parâmetros leucocitários e de proteínas de fase aguda na pododermatite infecciosa ovina. Pesq. Vet. Bras. 32(12):1289-1296. <http://dx.doi. org/10.1590/S0100-736X2012001200013>

Cavalcanti A.S.R., Aguiar G.M.N. \& Ayres M.C.C. 2004. Frequência da pododermatite em ovinos criados na microrregião dos Tabuleiros Costeiros no Estado da Bahia. Anais XXXI Congresso Brasileiro de Medicina Veterinária, São Luís, MA. (Abstract)

Gargano R.G., Benesi F.J., Birgel Junior E.H., Libera A.M.M.P.D., Gregory L., Sucupira M.C.A., Ortolani E.L., Gomes V. \& Pogliani F.C. 2013. Estudo retrospectivo das afecções locomotoras em ruminantes atendidos na Faculdade de Medicina Veterinária e Zootecnia da Universidade de São Paulo entre 2000 e 2012. Braz. J. Vet. Res. Anim. Sci. 50(4):286-293. <http:// dx.doi.org/10.11606/issn.2318-3659.v50i4p286-293>

Ghimire S.C., Egerton J.R. \& Dhungyel O.P. 1999. Transmission of virulent foot rot between sheep and goats. Aust. Vet. J. 77(7):450-453. <http:// dx.doi.org/10.1111/j.1751-0813.1999.tb12091.x> <PMid:10451731>

Hodgkinson 0. 2010. The importance of feet examination in sheep health management. Small Rumin. Res. 92(1-3):67-71.<http://dx.doi.org/10.1016/j. smallrumres.2010.04.007>

INEMA 2016. Relatório de informações hidrológicas e meteorológicas Instituto do Meio Ambiente e Recursos Hídricos. Disponível em <http:// monitoramento.inema.ba.gov.br \planilhas \plus \> Acesso em 10 out. 2016.

Lovatt F.M. 2015. Causes, control and costs of lameness in sheep. Vet. J. Ireland. 5(4):185-188. 
Maes D. \& Opsomer G. 2013. Lameness and claw lesions in sows, cows and small ruminants. Livest. Sci. 156(1/3):1. <http://dx.doi.org/10.1016/j. livsci.2013.06.001>

Morão R.P., Almeida A.C., Santos C.A., Oliveira F.D., Costa L.F. \& Carvalho Júnior I.S. 2015. Doenças infecciosas em criatórios de ovinos nas mesorregiões norte de Minas e Vale do Jequitinhonha, Minas Gerais, Brasil. Cader. Ciênc. Agrár. 7:63-74.

O’Leary C. 2014. Eradication and control of lameness in sheep. Vet. Ireland J. 4(7):377-379.

Olechnowicz J. \& Jaśkowski J.M. 2011. Lameness in small ruminants. Med. Vet. 67(11):715-719.

Pinheiro R.R., Gouveia A.M.G., Alves F.S.F. \& Haddad J.P.A. 2000. Aspectos epidemiológicos da caprinocultura cearense. Arq. Bras. Med. Vet. Zootec 52(5):534-543. <http://dx.doi.org/10.1590/S0102-09352000000500021>

Raadsma H. \& Egerton J. 2013. A review of footrot in sheep: Aetiology, risk factors and control methods. Livest. Sci. 156(1/3):106-114.<http://dx.doi. org/10.1016/j.livsci.2013.06.009>

Rather M.A., Wani S.A., Hussain I., Bhat M.A., Kabli Z.A. \& Magray S.N. 2011 Determination of prevalence and economic impact of ovine footrot in central Kashmir India with isolation and molecular characterization of Dichelobacter nodosus. Anaerobe 17(2):73-77.<http://dx.doi.org/10.1016/j. anaerobe.2011.02.003> <PMid:21397709>

Ribeiro L.A.0. 2010. Controle de Footrot (Podridão dos cascos). Disponível em <http://www.iz.sp.gov.br/pdfs/1284382639.pdf> Acesso em 15 out. 2016.
Riet-Correa F. 2007. Abscesso de pé, p.199-201. In: Riet-Correa F., Schild A.L., Lemos R.A.A. \& Borges J.R.J. (Eds), Doenças de Ruminantes e Equídeos. Vol.1. $3^{\text {a }}$ ed. Pallotti, Santa Maria.

Rodrigues C.A., Mendes L.C.N., Peiró J.R. \& Feitosa F.L.F. 2001. Ocorrência de um surto de "footrot" em rebanho de ovinos na região de Araçatuba, SP, Brasil. Revta Educ. Contin. CRMV-SP 4(3):12-19.

Silveira C.S., Damboriarena P.A., Morais R.M., Trost M.E., Pozzobon R. \& Anjos B.L. 2016. Lesões podais em ovinos da Mesorregião Sudoeste do Rio Grande do Sul. Pesq. Vet. Bras. 36(10):971-978. <http://dx.doi.org/10.1590/ s0100-736x2016001000009>

Smith E.M., Green O.D., Calvo-Bado L.A., Witcomb L.A., Grogono-Thomas R., Russell C.L., Brown J.C., Medley G.F., KilBride A.L., Wellington E.M. \& Green L.E. 2014. Dynamics and impact of footrot and climate on hoof horn length in 50 ewes from one farm over a period of 10 months. Vet. J. 201(3):295301. <http://dx.doi.org/10.1016/j.tvjl.2014.05.021><PMid:24973007>

Tadich N. \& Hernandez M. 2000. Prevalencia de lesiones podales en ovinos de 25 exploraciones familiares de la provincia de Valdivia, Chile. Arch. Med. Vet. 32(1):63-74. <http://dx.doi.org/10.4067/S0301-732X2000000100008>

Winter A.C. 2008. Lameness in sheep. Small Rumin. Res. 76(1/2):149-153. <http://dx.doi.org/10.1016/j.smallrumres.2007.12.008>

Winter J.R., Kaler J., Ferguson E., Kilbride A.L. \& Green L.E. 2015. Changes in prevalence of, and risk factors for, lameness in random samples of English sheep flocks: 2004-2013. Prev. Vet. Med. 122(1/2):121-128. <http://dx.doi. org/10.1016/j.prevetmed.2015.09.014> <PMid:26435034> 